\title{
USO Y FLORA LEÑOSA ASOCIADA A OECOPETALUM MEXICANUM (ICACINACEAE): UnA ESPECIE COMESTIBLE NATIVA DE LA SieRra de Misantla, Veracruz, México
}

\author{
Maite Lascurain-Rangel ${ }^{1,6}$, Sergio Avendaño-Reyes², Citlalli López-Binnqüist ${ }^{3}$, \\ Juan Carlos lópez-Acosta ${ }^{3}$, Melissa Covarrubias-Báez ${ }^{4}$ y Rodrigo Duno-de Stefano 5 \\ ${ }^{1}$ Red Ambiente y Sustentabilidad y ${ }^{2}$ Herbario XAL, Instituto de Ecología, A.C., Xalapa, \\ ${ }^{3}$ Centro de Investigaciones Tropicales, Universidad Veracruzana. Xalapa, Veracruz, México \\ ${ }^{4}$ Instituto de Investigaciones Forestales, Universidad Veracruzana. Xalapa, Veracruz, México \\ ${ }^{5}$ Centro de Investigación Científica de Yucatán, A.C. Mérida, Yucatán, México \\ ${ }^{6}$ Autor para la correspondencia maite.lascurain@inecol.edu.mx
}

\begin{abstract}
Resumen: En el presente estudio se describen los sitios de manejo y la flora leñosa asociada a Oecopetalum mexicanum (cachichín) en la Sierra de Misantla, Veracruz; así como la recolección, el uso y la estimación de los frutos con base en muestreos de vegetación, entrevistas y observaciones de campo. El cachichín crece en cuatro tipos de manejo: selva, cachichinal (selva dominada por O. mexicanum), cafetal y huerto. Los muestreos de vegetación se llevaron a cabo en seis parcelas distribuidas en los primeros tres tipos de manejo, en una superficie total de 0.3 ha, fueron considerados todos los individuos $\geq 1 \mathrm{~cm}$ a la altura del pecho. En los mismos sitios se estimó la producción de frutos de 60 árboles. Debido a la reducida extensión de los huertos, se realizaron ocho muestreos florísticos independientes. La riqueza florística en conjunto fue de 79 especies pertenecientes a 35 familias distribuidas de la siguiente forma: 52 en la selva, 27 en el cachichinal y 28 en el cafetal. En los huertos se registró la presencia de 11 especies dominantes. La fisionomía en las selvas no se ve afectada por la recolección del cachichín. Los cachichinales conservan los atributos fisionómicos similares a las selvas con mayor abundancia de especies útiles nativas; en el cafetal, hay presencia de especies exóticas y pocos individuos de cachichín. La producción promedio anual fue de 680 frutos/árbol ( \pm 167.4 D.E.), sin encontrar diferencias entre los distintos tipos de manejo $(P=0.75)$. El cachichín se recolecta ampliamente en la región para autoconsumo y para su venta, comercializándose en la Sierra y en ciudades como Misantla y Xalapa.
\end{abstract}

Palabras clave: cachichín, conservación vegetal, frutos comestibles silvestres.

\begin{abstract}
We evaluated the use of the Mexican tree species Oecopetalum mexicanum (cachichín) in the Sierra de Misantla, Veracruz, Mexico. Based on vegetation samples, interviews and field observations, we describe the woody plants associated with this species, management sites and the collection and use of the edible fruit. The cachichín grows under four types of management: natural forest, cachichinal (O. mexicanum dominated forest), coffee plantation and orchard. Vegetation was sampled in six plots representing the first three management types, comprising a total area $0.3 \mathrm{ha}$, and including all individuals $\geq 1 \mathrm{~cm}$ diameter at breast height. Fruit production was estimated in 60 trees at the same sites. Due to the limited area of the orchards, eight independent floristic samples were taken. Total richness was 79 species from 35 families, distributed as follows: natural forest (52), cachichinal (27) and coffee plantation (28). The orchards presented eleven dominant species. Physiognomy in the forests was unaffected by cachichín collection. Cachichinal retained physiognomic attributes that were similar to that of the forests, but with an abundance of useful native species. The coffee plantation featured exotic species with few cachichín individuals. Annual average fruit production was 680 ( \pm 167.4$)$ fruits/tree and did not differ significantly between management types $(P=0.75)$. Cachichín is widely collected in the region for direct consumption and sale in the Sierra and in cities such as Misantla and Xalapa.
\end{abstract}

Key words: cachichín, plant conservation, wild edible fruits.

$\mathbf{L}$ a interacción entre los humanos y los bosques puede ser de diversos de tipos, desde la recolección de sus productos útiles, la protección de árboles tanto en los bosques naturales o secundarios, hasta su cultivo (Wiersum, 1997a, b; Simons y Leakey, 2004; Belcher et al., 2005). Ningu- na de estas formas de interacción ocurre como un proceso unidireccional o irreversible (Michon y De Foresta, 1997; Wiersum, 1997a); de tal manera que en muchas áreas, una especie de árbol útil puede crecer en diferentes tipos de bosques y/o sistemas de cultivo o silvopastoriles, y coexistir 
conformando paisajes específicos (Wiersum, 1997a; Schroth et al., 2004; Michon et al., 2007).

La diversidad vegetal de un área puede ser modificada como consecuencia del aprovechamiento de una o varias especies y por la intensidad de su manejo. Cualquier tipo de aprovechamiento tiene un impacto ecológico y éste depende del tipo de vegetación, de la intensidad de la práctica de manejo, de la especie y de la parte de la planta que se utiliza (Peters, 1996; Wong et al., 2001). Por lo tanto, evaluar el aprovechamiento de una especie puede ayudar a identificar los cambios en los procesos ecosistémicos (Ticktin y Shackleton, 2011) y en la modificación del paisaje.

La Sierra de Misantla, también conocida como Sierra de Chiconquiaco, se ubica en el centro del estado de Veracruz, se reconoce como una zona de origen totonaco (Kelly y $\mathrm{Pa}$ lerm, 1952), de alta riqueza florística y "crítica en la distribución de muchos géneros arbóreos tropicales, principalmente de las regiones de baja altitud en América tropical" (Gómez-Pompa, 1966). En esta región se encuentra Oecopetalum mexicanum Greenm. \& C.H.Thomps., árbol nativo que produce semillas comestibles. Tanto el árbol como la semilla se conocen con el nombre de cachichín, palabra de origen totonaco que significa fruto amargo.

Gutiérrez (1994) describió Oecopetalum mexicanum como un árbol de 2-25 m de altura, con ramas glabras o pubescentes; de hojas simples, pecioladas, haz verde obscuro, envés verde claro, elíptico-lanceoladas, de $15-25 \mathrm{~cm}$ de largo, de 7-10 cm de ancho, glabras, margen entero, ápice apiculado; inflorescencias de tipo terminal o axilar, flores con cinco pétalos blancos y anteras amarillas; el fruto, una drupa verde de color marrón (al madurar), globosa, rugosa, glabra de 2 a $3 \mathrm{~cm}$ de largo, 1 a $2 \mathrm{~cm}$ de ancho; semilla de color blanco (Figura 1A-C). Oecopetalum mexicanum prospera en suelos húmedos y laderas pronunciadas. Su distribución natural en la Sierra de Misantla va desde los 400 hasta los 1,100 m s.n.m. En esa franja se puede observar como especie primaria en poblaciones distribuidas en ecotonos de selva baja caducifolia y selva alta subperennifolia, selva mediana perennifolia, selva alta perennifolia y subperennifolia
(Gutiérrez, 1993), esta última referida por Gómez-Pompa (1966) como selva de lauráceas. También crece en fincas de café de sombra, en solares o huertos de las casas y en una asociación vegetal llamada "cachichinal", cuyo componente dominante es $O$. mexicanum. Este tipo de manejo se establece y se reproduce a través del tiempo dentro de la estructura original del bosque y no está asociada con la producción agrícola (Covarrubias-Báez, 2009). En este estudio se presenta una descripción de los sitios de manejo y la diversidad vegetal asociada a $O$. mexicanum, la recolección y el uso de los frutos comestibles, así como la estimación de la producción de frutos.

\section{Material y método}

Área de estudio. La Sierra de Misantla está conformada por un complejo de montañas que comprende los municipios de Acatlán, Chiconquiaco, Landero y Coss, Miahuatlán, Misantla, Tenochtitlán, Tlacolulan, Tonayán y Yecuatla (Gutiérrez, 1993). Se ubica en las coordenadas $19^{\circ} 52^{\prime}$ y $21^{\circ}$ 02' latitud norte y $96^{\circ} 46^{\prime}$ y $97^{\circ} 59^{\prime}$ longitud oeste (Figura 2). Los elementos más distintivos son: cultivos de café, maíz y cítricos, y parches de selvas en diferentes estados de conservación, tanto de selva baja caducifolia como de selva alta perennifolia y en ecotonía entre estos dos ecosistemas (Gómez-Pompa, 1966; Gutiérrez, 1993). Un estudio etnobotánico indica que en el municipio de Misantla, el de mayor superficie de la Sierra, hay 372 especies vegetales útiles (Ambrosio y Avendaño, 1999).

Este estudio se llevó a cabo en dos comunidades de la Sierra de Misantla: Pueblo Viejo (municipio de Misantla) y Roca de Oro (municipio de Yecuatla), con 847 y 443 habitantes, respectivamente, con la finalidad de conocer tanto las prácticas de manejo relacionadas con Oecopetalum mexicanum, como los aspectos más importantes acerca de la recolección de sus frutos, la temporalidad, los volúmenes recolectados, los sitios de recolección, los usos y el consumo. Se obtuvo información adicional mediante recorridos y observaciones de campo en compañía de recolectores y

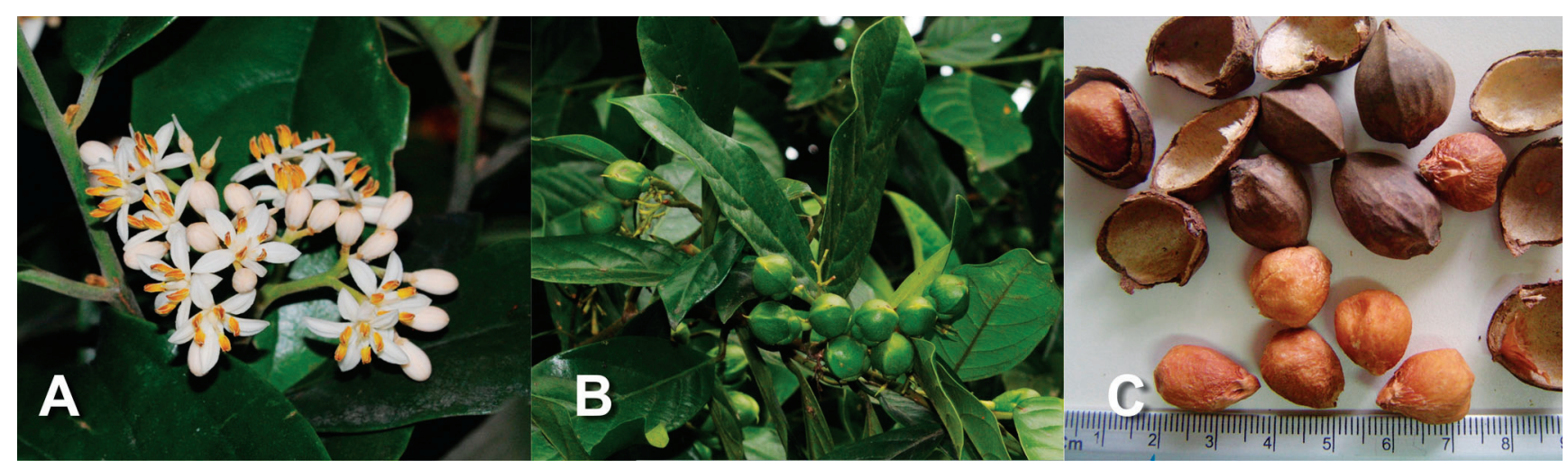

Figura 1. A. flores, B. semillas, C. semillas tostadas. 


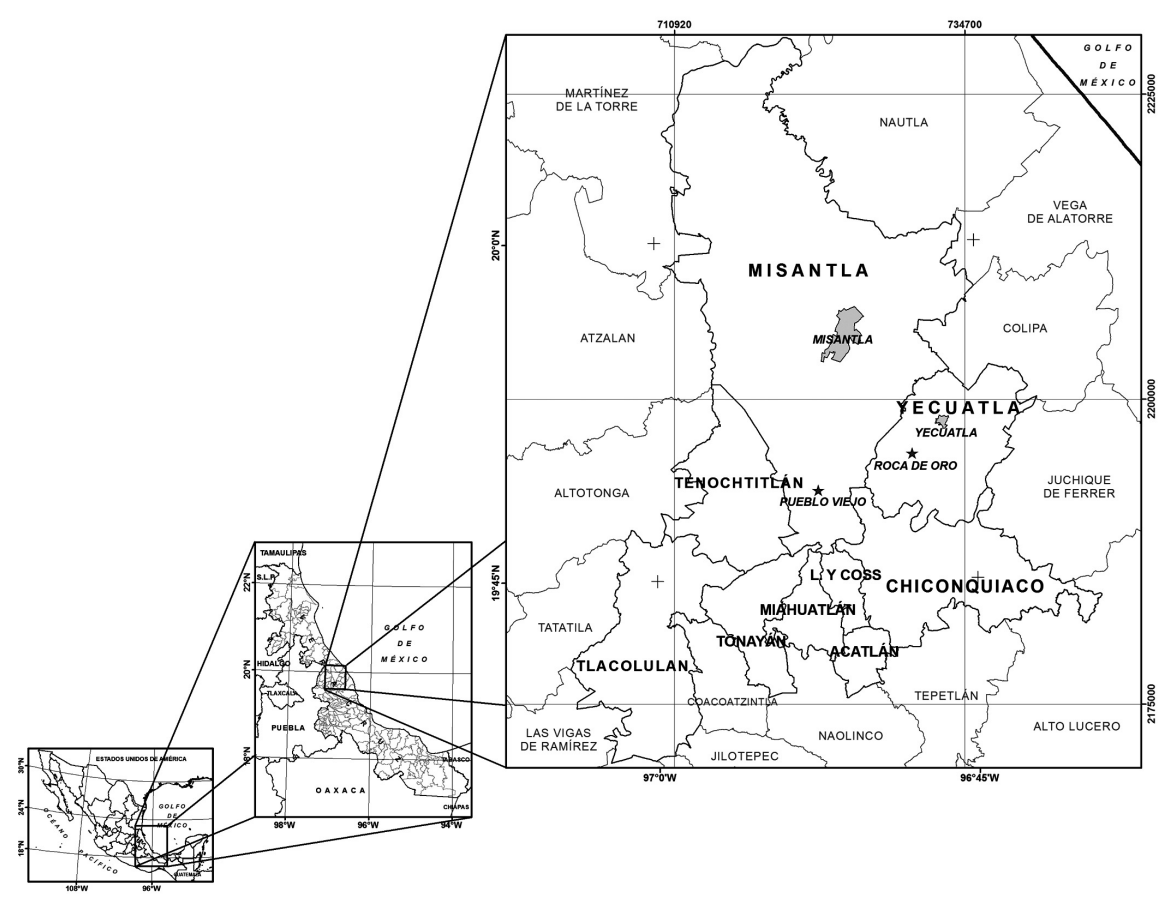

Figura 2. Mapa de localización de la Sierra de Misantla, Veracruz, México.

procesadores del cachichín. También se aplicaron 75 entrevistas semiestructuradas (Alexiades, 1996; Martin, 2000; Bernard, 2006): 25 en Roca de Oro y 50 en Pueblo Viejo.

Para identificar las principales especies vegetales asociadas a los tipos de manejo donde se encuentra Oecopetalum mexicanum, se seleccionaron seis parcelas distribuidas en tres tipos de manejo: dos en bosque (ecotonía entre selva baja caducifolia y selva alta subperennifolia), dos en cachichinal (bosque dominado por $O$. mexicanum) y dos en cafetal. En cada tipo de manejo se estudió una superficie total de 0.1 ha y se muestreó la diversidad de especies, para ello se siguió el método propuesto por Gentry $(1982,1988)$, el cual consiste en establecer al azar diez transectos de $50 \mathrm{~m}$ de longitud, incluyendo un metro de cada lado $\left(100 \mathrm{~m}^{2}\right)$. En cada transecto se identificaron las especies leñosas y se midió el diámetro a la altura del pecho (DAP); se tomaron en cuenta los individuos que a $1.30 \mathrm{~m}$ de altura presentaran un diámetro mayor o igual a $1 \mathrm{~cm}$.

Otro sitio en donde tradicionalmente se mantiene el cachichín son los huertos; sin embargo, dada su limitada extensión sólo se tomaron datos en ocho de ellos para acumular 0.1 ha y hacerlos equiparables con los datos de otros tipos de manejo. La estimación de la producción de frutos de Oecopetalum mexicanum se hizo mediante la selección de 60 árboles con un DAP promedio de $15.8 \mathrm{~cm}(+/-7.5$ D.E), distribuidos en los tres sitios de manejo (bosque, cachichinal y cafetal, $15 \mathrm{c} / \mathrm{u}$ ). En cada sitio se marcaron y embolsaron diez panículas por árbol, que de acuerdo con criterios filotáxicos debe hacerse en ramas de segundo o tercer nivel. Posteriormente, se cuantificó el número total de panículas por árbol; para ello se utilizó la relación, número de panículas por número promedio de frutos por panícula como un estimador del total de frutos por árbol.

\section{Resultados}

Uso y recolección. La semilla de cachichín tiene un sabor amargo, el aroma y textura es similar al cacahuate; su aspecto es semejante al de una nuez de dos valvas que se rompe con los dientes para su consumo. Se consume a cualquier hora del día, por lo general tostado y en ocasiones hervido. Es una semilla muy apreciada por los habitantes de la Sierra de Misantla; generalmente se ofrece como cortesía a las visitas, amigos y parientes. Las entrevistas revelaron que en ciertos casos se envían paquetes de cachichín tostado a los parientes que viven en otros sitios del país e incluso a Estados Unidos, reforzando así la identidad regional por este recurso. La principal forma de venta del cachichín es verde o seco y se circunscribe a las comunidades de la Sierra; también se expende tostado en los mercados ambulantes y en las calles de ciudades como Naolinco, Misantla y Xalapa (Lascurain et al., 2009).

Además del uso comestible, los habitantes de la zona atribuyen al cachichín propiedades medicinales para curar enfermedades del hígado, diabetes y la vesícula biliar. La madera de árboles caídos, enfermos, viejos o producto de podas, es utilizada para la construcción de viviendas y como leña. Se ha registrado que, en condición verde, la madera de cachichín tiene resistencia similar a la del pino (Lascurain et al., 2007). Otro uso es la elaboración 


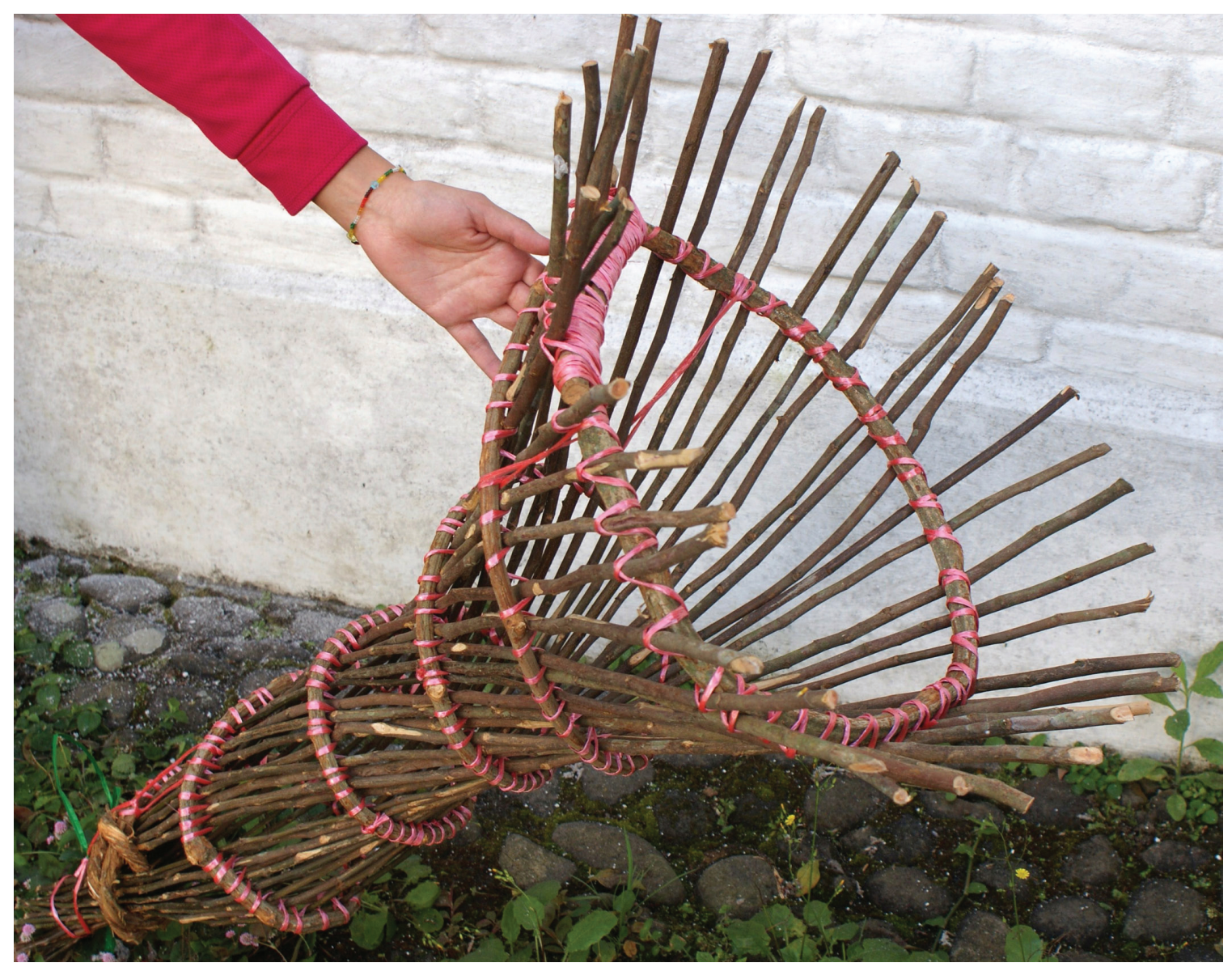

Figura 3. Canasta para atrapar langostinos hecha con varas de Oecopetalum mexicanum.

de canastas en forma de cono que sirven para capturar langostinos, éstas son elaboradas con las ramas nuevas de los árboles (Figura 3).

La recolección del cachichín se lleva a cabo durante los meses de abril y mayo. Los frutos se recogen del suelo, se buscan entre la hojarasca, a veces con ayuda de una varita o directamente con las manos. En ciertos casos la gente barre la hojarasca del cachichinal con escobas hechas de ramas o varas secas para facilitar la recolección. En las localidades estudiadas, los pobladores se organizan en grupos (familiares, amigos y vecinos) para desarrollar esta actividad y de acuerdo con los entrevistados, es una buena oportunidad para la convivencia y el entretenimiento; los días y el tiempo que invierten dependen de las habilidades de cada recolector y del destino final del producto. La recolección se inicia en la madrugada o en las primeras horas del día y puede durar de tres a siete días por semana. Una persona puede recoger de 7 a $30 \mathrm{~kg}$ de cachichín por día, en tanto grupos de dos o tres personas pueden cosechar entre 30 y $100 \mathrm{~kg}$ en jornadas largas. Las entrevistas indican que una familia de tres a cuatro miembros puede recoger aproximadamente 500 kg de cachichín en 15 a 20 días.

Se observó que en algunas comunidades de la Sierra, y en ciertas épocas del año, el ingreso por la venta del cachichín puede superar a la del café; por ejemplo, en 2008 el kilogramo de café cereza se vendía a cinco pesos y el de cachichín verde a diez pesos y en ese año el precio por kilo de fruto tostado se llegó a cotizar en cien pesos en la ciudad de Xalapa. Aproximadamente $100 \mathrm{~g}$ de cachichín tostado cuesta de diez a quince pesos; cabe destacar este dato, ya que durante la temporada de recolección de cachichín no se cosecha café. A pesar del beneficio monetario y el valor de uso asociado en los diferentes tipos de manejo del cachichín observados en este trabajo, aún está pendiente por realizar un análisis específico del valor económico y de autoconsumo a nivel familiar, que incluya entre otros aspectos, la tenencia de la tierra, insumos y mano obra, cuyo método y marco conceptual están fuera del propósito de este trabajo.

Flora leñosa asociada. En los cuatro diferentes tipos de manejo donde se desarrolla Oecopetalum mexicanum (bosque, cachichinal, cafetal y huerto), se registraron 79 especies (nativas e introducidas) de 35 familias. Las familias mejor representadas fueron Fabaceae con ocho especies y Euphorbiaceae con cinco, seguidas de Lauraceae, Rubiaceae y Rutaceae con cuatro especies cada una; el resto oscila entre una 
Oecopetalum mexicanum, fruto comestible de la Sierra de Misantla, VeracruZ.

Cuadro 1. Especies más abundantes por tipo de manejo asociadas a Oecopetalum mexicanum en la Sierra de Misantla, Veracruz.

\begin{tabular}{|c|c|c|c|c|c|}
\hline Familia & Nombre científico & Selva & Cachichinal & Cafetal & Huerto \\
\hline Adoxaceae & Sambucus nigra L. & & & $X$ & \\
\hline Araliaceae & Oreopanax capitatus (Jacq.) Decne. \& Planch. & $X$ & & & \\
\hline \multirow[t]{2}{*}{ Arecaceae } & Chamaedorea alternans H.Wendl. & & $X$ & & \\
\hline & Chamaedorea oblongata Mart. & & $x$ & & \\
\hline Asteraceae & Vernonia patens Kunth & & & $X$ & \\
\hline Cannabaceae & Trema micrantha (L.) Blume & & $X$ & & \\
\hline Capparaceae & Capparis mollicella Standl. & $X$ & & & \\
\hline Caricaceae & Carica papaya L. & $X$ & & & $\mathrm{X}$ \\
\hline Chrysobalanaceae & Couepia polyandra (Kunth) Rose & & & & $x$ \\
\hline \multirow[t]{5}{*}{ Euphorbiaceae } & Alchornea latifolia Sw. & $X$ & & & \\
\hline & Cnidoscolus multilobus (Pax) I.M.Johnst. & $x$ & & & \\
\hline & Croton draco Schltdl. \& Cham. & $X$ & & & \\
\hline & Ricinus communis $\mathrm{L}$. & & & $X$ & \\
\hline & Sapium lateriflorum Hemsl. & $X$ & & & \\
\hline \multirow[t]{4}{*}{ Fabaceae } & Inga semialata (Vell.) Mart. & & $X$ & $X$ & \\
\hline & Inga vera Willd. & & & $\mathrm{X}$ & \\
\hline & Inga jinicuil Schltdl. \& Cham. ex G.Don & & & & $\mathrm{X}$ \\
\hline & Inga paterno Harms & & & $\mathrm{X}$ & \\
\hline Icacinaceae & Oecopetalum mexicanum Greenm. \& C.H. Thomps. & $X$ & $X$ & $\mathrm{X}$ & $X$ \\
\hline Lamiaceae & Cornutia sp. & & & 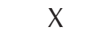 & \\
\hline \multirow[t]{3}{*}{ Lauraceae } & Beilschmiedia anay (S.F.Blake) Kosterm. & & $X$ & $\mathrm{X}$ & \\
\hline & Ocotea sp. & $X$ & & $\mathrm{X}$ & \\
\hline & Persea americana Mill. & & & & $X$ \\
\hline Meliaceae & Trichilia havanensis Jacq. & & $X$ & & \\
\hline \multirow[t]{2}{*}{ Moraceae } & Pseudolmedia glabrata (Liebm.) C.C.Berg & $X$ & & & \\
\hline & Trophis racemosa (L.) Urb. & $X$ & $X$ & & \\
\hline Musaceae & Musa paradisiaca L. & & $x$ & $X$ & $X$ \\
\hline Primulaceae & Ardisia compressa Kunth & & & & $x$ \\
\hline \multirow[t]{3}{*}{ Rubiaceae } & Coffea arabica L. & & $X$ & $X$ & $\mathrm{X}$ \\
\hline & Psychotria chiapensis Standl. & $x$ & & & \\
\hline & Psychotria flava Oerst. ex Standl. & $x$ & & & \\
\hline \multirow[t]{2}{*}{ Rutaceae } & Citrus spp. & & & & $X$ \\
\hline & Citrus tangerina Yu. Tanaka & & & $X$ & \\
\hline \multirow[t]{2}{*}{ Salicaceae } & Pleuranthodendron lindenii (Turcz.) Sleumer & $X$ & & & \\
\hline & Xylosma flexuosa (Kunth) Hemsl. & & & & $X$ \\
\hline \multirow[t]{2}{*}{ Sapindaceae } & Cupania glabra Sw. & $X$ & & & \\
\hline & Cupania dentata DC. & & & $X$ & \\
\hline Solanaceae & Capsicum sp. & & & & $X$ \\
\hline Urticaceae & Urera caracasana (Jacq.) Gaudich. ex Griseb. & $X$ & & & \\
\hline
\end{tabular}

y tres especies en cada familia. En el cuadro 1 se mencionan las especies más abundantes en los distintos tipos de manejo estudiados.

Selva o monte.- Corresponde a fragmentos de vegetación en ecotonos de selva baja caducifolia y de selva alta subperennifolia, donde crece el cachichín de manera natural y de forma abundante; se cuantificaron 377 individuos en 0.1 ha, casi el $50 \%$ son menores a $1.5 \mathrm{~cm}$ de DAP; se registraron 52 taxa. Las especies importantes en términos de abundan- cia fueron Carica papaya y Pseudolmedia glabrata, de las cuales se registraron 134 y 91 individuos, respectivamente. Un componente fisionómico destacado en las selvas de la zona es Ocotea sp., la cual mostró una alta densidad (82 individuos) y dominancia ( $60 \%$ de la total) en 0.1 ha, ya que muchos de estos árboles presentan tallas superiores a un metro de DAP. Otras especies importantes en las selvas, en términos de abundancia ( $\geq$ diez individuos) son: Alchornea latifolia, Capparis mollicella, Chamaedorea alternans, Cnidoscolus multilobus, Croton draco, Cupania glabra, 
Oreopanax capitatus, Pleuranthodendron lindenii, Psychotria chiapensis, P. flava, Sapium lateriflorum, Trophis racemosa y Urera caracasana.

Cachichinal.- Está caracterizado principalmente por la abundancia de Oecopetalum mexicanum, aunque se encuentran otras especies útiles. Los cachichinales son especialmente cuidados y manejados por los dueños de las tierras mediante prácticas de manejo que favorecen su crecimiento, tales como la eliminación de malezas y árboles viejos, podas, etc. En este tipo de manejo se registró una menor riqueza florística (27 especies) y $O$. mexicanum es la especie más representativa con 143 ind./0.1 ha. Otro componente importante es Coffea arabica, con un total de 90 ind./0.1 ha. Además, se encuentran algunas especies bien representadas con diez o menos individuos, entre ellas: Beilschmiedia anay, Chamaedorea alternans, C. oblongata, Inga vera, Musa paradisiaca, Trema micrantha, Trichilia havanensis y Trophis racemosa.

Cafetal.- Tanto en Roca de Oro como en Pueblo Viejo, el policultivo tradicional de café bajo sombra es común; posee una alta diversidad de especies introducidas que cuentan con usos locales, por ejemplo: medicinales, alimenticios y maderables. El cachichín se tolera, aunque en baja densidad; eventualmente se podan sus ramas bajas. Además de la alta densidad de Coffea arabica (95 ind./0.1 ha) se encontraron 28 especies, entre ellas: Inga paterno, I. semialata e I. vera, que en conjunto muestran una densidad de 26 ind./0.1 ha. Otras especies representadas con diez o menos individuos son: Beilschmiedia anay, Cornutia sp., Citrus tangerina, $\mathrm{Cu}$ pania dentata, Musa paradisiaca, Ricinus communis, Sambucus nigra y Vernonia patens. Aquí solo se registraron dos individuos de Ocotea sp. de gran tamaño (DAP > $1.5 \mathrm{~m}$ ).

Huerto.- En este tipo de manejo se mantienen de uno a cinco árboles de cachichín que han sido introducidos mediante semillas o plántulas. Se registraron 15 especies asociadas, donde las comestibles son las más abundantes como Ardisia compressa, Capsicum sp., Carica papaya, Citrus spp., Coffea arabica, Couepia polyandra, Inga jinicuil, Musa paradisiaca, Persea americana y Xylosma flexuosa.

Producción de frutos. Del total de 60 árboles analizados con un DAP promedio de $15.8 \mathrm{~cm}$ (+/- 7.5 D.E.), las estimaciones señalan que un árbol reproductivo desarrolla en promedio alrededor de 1,720 panículas ( \pm 562 D.E.), con una producción promedio de 0.5 frutos por panícula $( \pm 0.19$ D.E.); esto da como resultado una producción de 680 frutos por árbol ( \pm 167.4 D.E.), lo que equivale aproximadamente a un poco más de un kilogramo de cachichín fresco por árbol. No se encontraron diferencias significativas en la producción de frutos dependiendo del tipo de manejo $\left(\mathrm{F}_{3,56}=\right.$ $0.402, P=0.75)$.

\section{Discusión}

Diversos estudios de especies arbóreas mexicanas con frutos comestibles muestran algunas similitudes en el manejo, aprovechamiento y autoconsumo con Oecopetalum mexicanum, por ejemplo: Beilschmiedia anay, Brosimum alicastrum (Peters, 1989), Byrsonima crassifolia (Martínez, 2008), Inga jinicuil, Persea schiedeana (Cruz-Castillo et al., 2007; Bost, 2009) y Pouteria sapota. La comercialización en general de estas especies es regional, a excepción del fruto de $P$. sapota que es muy conocido en el país y tiene un mercado que va más allá de su área de distribución (Nava-Cruz y Ricker, 2004). Brosimum alicastrum actualmente tiene una amplia difusión para su cultivo y formas de uso (Meiners et al., 2009). Algunas especies muestran una disminución en sus poblaciones en ciertas áreas geográficas; esto se debe entre otras causas, al cambio de uso del suelo, tal es el caso de B. anay (EscamillaPrado y Robledo-Martínez, 1996), B. alicastrum (Meiners et al., 2009) e I. jinicuil (Pulido-Salas, 2009).

La información recopilada para el cachichín muestra que las actividades de recolección y consumo de estos frutos forman parte de los mecanismos que contribuyen al mantenimiento de los lazos de convivencia familiar y de amistad entre vecinos. La información reunida también permite reconocer las relaciones que existen entre las actividades y los sitios de recolección, en donde la distancia, la facilidad y el volumen potencial para recolectar forman parte de los principales criterios para decidir y organizar la forma de recolección del cachichín.

La selva es uno de los sitios preferidos por los recolectores debido a la abundancia de los árboles de esta especie, a pesar de que la distancia que hay recorrer es mayor, comparada con los otros sitios de manejo. La distancia que caminan los recolectores en promedio es de uno a cinco kilómetros; el tiempo de recorrido es variable, ya que depende de la edad de la persona, del clima y de las condiciones del camino. Las selvas son los sitios que presentan una mayor riqueza de plantas, dominada por individuos adultos de Ocotea sp. y Pseudolmedia glabrata (Covarrubias-Báez, 2009). Estos datos son compatibles con la descripción de la vegetación mencionada anteriormente por Gómez-Pompa (1966). Actualmente en las selvas de la región es posible observar diversos claros, tanto naturales como veredas abiertas por los usuarios; esos espacios son dominados por Carica papaya y Cnidoscolus multilobus. En las selvas, los árboles de $O$. mexicanum no reciben ninguna práctica de manejo, únicamente los pobladores se limitan a la recolecta del fruto.

En el cachichinal se observa la dominancia de Oecopetalum mexicanum; en estos sitios se eliminan algunos elementos de la vegetación competitiva para favorecer su crecimiento. Así, gran parte del manejo está dirigido a aumentar la producción y a mejorar las condiciones de la recolección; además se permite la presencia de otras especies útiles, localmente apreciadas, como Chamaedorea spp. y Trichilia 
havanensis. Cabe destacar que su fisionomía (en términos de estructura y apertura del dosel), es similar a los bosques originales, lo cual, a reserva de realizar otros estudios, los convierte en zonas de resguardo de especies con valor cultural y ecológico. Es posible que este tipo de manejo vaya en aumento, ya que de acuerdo con los cachichineros: "ahora es mejor sembrar cachichín que café; cada día es más conocido por la gente y vale más". La distancia entre los asentamientos humanos y los cachichinales es más corta que la de los bosques; por lo tanto, durante la recolección es uno de los sitios igualmente frecuentados.

Por otra parte, en los cafetales se toleran pocos árboles de cachichín, los dueños de las parcelas no permiten que se multipliquen de manera masiva porque afirman que "secan" las plantas de ese cultivo. Finalmente, en los huertos se mantienen pocos árboles de Oecopetalum mexicanum, principalmente por falta de espacio, ya que existen otros árboles útiles y plantas de ornato. Tanto el cafetal como el huerto son los sitios que presentan la mayor facilidad para la recolección de frutos, dada la cercanía con las viviendas.

Los aspectos ecológicos y de manejo de la selva y cachichinal indican "altos índices de valores de importancia para la regeneración de Oecopetalum mexicanum, 42.37 y $67.97 \%$ respectivamente" (Covarrubias-Báez, 2009). Las distintas formas de uso y aprovechamiento del cachichín en el área de estudio posibilitan la conservación de diversas especies útiles y ejemplifican la interacción entre el ser humano y el recurso, expresada desde la recolección del fruto en los bosques hasta el manejo especializado (cachichinal).

El impacto del aprovechamiento del cachichín en la vegetación de la sierra de Misantla se refleja principalmente en los cachichinales. Las actuales observaciones indican que el bosque mantiene su fisionomía original debido a que únicamente es un sitio de recolección; en el cafetal las prácticas de manejo están enfocadas en asegurar la producción de café y la presencia de árboles de cachichín es escasa. El cachichinal presenta una fisionomía similar a la del bosque, donde se favorecen las especies locales útiles sin perjudicar la abundancia de la especie de uso principal (Oecopetalum mexicanum) que le da el nombre a estos parches.

Este trabajo permite destacar la importancia ecológica, social y económica del cachichín en la Sierra de Misantla. Se reconoce su amplio uso en dicha área, tanto para el autoconsumo como para la venta; lo cual indica una constante motivación entre los habitantes para mantener esta especie en los diferentes tipos de manejo. Lo anterior sugiere, a reserva de mayores estudios, que el cachichín es un recurso biocultural que se maneja y aprovecha de manera sustentable.

\section{Agradecimientos}

Nuestro agradecimiento a los revisores por sus valiosas sugerencias para mejorar este trabajo. A la Comisión Nacional Forestal por el apoyo financiero del Fondo Sectorial
Conafor-Conacyt 2002-C01-6510 y del Programa Pro-árbol S20073000662. A Rafael Villegas y César Gallo por la elaboración del mapa. A las familias López y Triano por las facilidades ofrecidas durante el desarrollo de este estudio.

\section{Literatura citada}

Alexiades M.N. 1996. Selected Guidelines for Ethnobotanical Research: A Field Manual. The New York Botanical Garden, Nueva York.

Ambrosio M.M. y Avendaño R.S. 1999. Catálogo de plantas útiles del municipio de Misantla, Veracruz. La Ciencia y el Hombre 31:43-87.

Belcher B., Michon G, Angelsen A, Ruiz P.M. y Asbjornsen H. 2005. The socioeconomic conditions determining the development, persistence, and decline of forest garden systems. Economic Botany 59:245-253.

Bernard R.H. 2006. Research Methods in Anthropology, Qualitative and Quantitative Approaches. Altamira Press, Oxford.

Bost J.B. 2009. Edible plants of the Chinantla, Oaxaca, Mexico with an emphasis on the participatory domestication prospects of Persea schiedeana. Tesis maestría, Universidad de Florida, Gainesville, 113 pp.

Covarrubias-Báez M. 2009. Estructura y diversidad vegetal asociada a la presencia de Oecopetalum mexicanum Greenm. \& C.H. Thomps., (Icacinaceae) en tres sistemas de manejo de la sierra de Misantla, Veracruz, México. Tesis licenciatura, Facultad de Biología, Universidad Veracruzana, Xalapa, 50 pp.

Cruz-Castillo J.G., Del Ángel-Coronel O.A, de La Cruz-Medina, J. y Joaquín-Martínez M.C. 2007. Características morfológicas y bioquímicas de frutos de chinene (Persea schiedeana Nees.) Revista Chapingo Serie Horticultura 13:141-147.

Escamilla-Prado E. y Robledo-Martínez J.D. 1996. El anayo (Beilschmedia anay (Blake) Kosterm) recurso genético en Puebla y Veracruz. Revista de Geografía Agrícola 22-23:99-213.

Gentry A.H. 1982. Patterns of neotropical plant species diversity. En: Hecht M.K., Wallace B. y Prance E.T. Eds. Evolutionary Biology, Volume 15, pp. 1-84, Plenum Press, Nueva York.

Gentry A.H. 1988. Tree species richness of upper Amazonian forests. Proceedings of the National Academy of Sciences USA 85:156-159.

Gómez-Pompa A. 1966. Estudios Botánicos en la Región de Misantla, Veracruz. Instituto Mexicano de Recursos Naturales Renovables A.C., México, D.F.

Gutiérrez B.C. 1993. Listado Florístico de la Sierra del Chiconquiaco, Ver. Textos Universitarios. Universidad Veracruzana, Xalapa.

Gutiérrez C. 1994. Icacinaceae. En: Sosa V. y Gómez-Pompa A. Eds. Fascículo No. 80. Flora de Veracruz, pp 1-16, Instituto de Ecología, A.C. y University of California, Xalapa.

Kelly I.T. y Palerm A. 1952. The Tajin Totonac. Part I. History, Subsistence, Shelter and Technology. Volume 13 of Publications of the Institute of Social Anthropology. Smithsonian Institution. Washington, DC.

Lascurain M., Ángeles-Álvarez G., Ortega Escalona F., Ordoñez C.V.R., Ambrosio M. y Avendaño S. 2007. Características anatómicas y propiedades mecánicas de la madera de Oecopetalum mexicanum Greenm. \& C.H.Thomps. (Icacinaceae) de la sierra de Misantla, Veracruz, México. Madera y Bosques 13:83-95. 
Lascurain M., López C. y Zamora P. 2009. Production chain of Oecopetalum mexicanum (Cachichín): a tropical fruit tree from México. Acta Horticulturae 806:519-524.

Martin G. 2000. Etnobotánica. Manual de Métodos. Manuales de Conservación de la Serie Pueblos y Plantas. World Wildlife Fund, Organización de las Naciones Unidas para la Educación, la Ciencia y la Cultura, Royal Botanic Gardens, Nordan Comunidad. Montevideo.

Martínez M.E. 2008. El nanche (Byrsonima crassifolia (L.) H.B.K.), un recurso fitogenético subutilizado en México. En: Cruz C.J.G y Torres L.P.A. Coords. Enfoques Tecnológicos en la Floricultura, pp. 87-99, Universidad Autónoma Chapingo, Texcoco.

Meiners M., Sánchez Garduño C. y De Blois S. 2009. El ramón: Fruto de nuestra cultura y raíz para la conservación. Biodiversitas 87:7-10.

Michon G. y De Foresta H. 1997. Agroforests: pre-domestication of forest trees or true domestication of forest ecosystems? $\mathrm{Ne}$ therlands Journal of Agricultural Science 45:451-462.

Michon G., De Foresta H., Levang P. y Verdeaux F. 2007. Domestic forest: a new paradigm for integrating local communities' forestry into tropical forest science. Ecology and Society 12:1.

Nava-Cruz Y. y Ricker M. 2004. El Zapote Mamey [Pouteria sapota (Jacq.) H.E. Moore \& Stearn], un fruto de la selva mexicana con alto valor comercial. En: Alexiades M.N. y Shanley P. Eds. Productos Forestales, Medios de Subsistencia y Conservación: Estudios de Caso sobre Sistemas de Manejo de Productos Forestales No Maderables (Volumen 3, América Latina), pp. 43-62, Centro para la Investigación Forestal Internacional, Bogor Barat.

Peters C.M. 1989. Reproduction, growth and the population dynamics of Brosimum alicastrum Sw. in a moist tropical forest of central Veracruz, Mexico. Tesis doctoral, Yale University, New Haven, 258 pp.

Recibido: 23 de agosto de 2012

Aceptado: 14 de febrero de 2013
Peters C.M. 1996. The Ecology and Management of Non-timber Forest Resources. World Bank Technical Paper No. 322. World Bank, Washington, DC.

Pulido-Salas M.T.P. 2009. Inga jinicuil (“jinicuil”) en Coatepec, Ver., México, como árbol frutal nativo: potencial económico y factores que afectan su permanencia. Tesis doctoral, Colegio de Postgraduados, Campus Veracruz,Veracruz, 178 pp.

Schroth G., Harvey C.A. y Vincent G. 2004. Complex agroforests: Their structure, diversity, and potential role in landscape conservation. En: Schroth G., da Fonseca G.A.B., Harvey C.A., Gascon C., Vasconcelos H.L. e Izac A.N. Eds. Agroforestry and Biodiversity Conservation in Tropical Landscapes, pp. 227260, Island Press, Washington, DC.

Simons A.J. y Leakey R.R.B. 2004. Tree domestication in tropical agroforestry. Agroforestry Systems 61:167-181.

Ticktin T. y Shackleton C. 2011. Harvesting non-timber forest products sustainably: opportunities and challenges. En: Shackleton S., Shackleton C. y Shanley P. Eds. Non-Timber Forest Products in the Global Context, pp. 149-169, Springer-Verlag, Berlín.

Wiersum K.F. 1997a. Indigenous exploitation and management of tropical forest resources: an evolutionary continuum in forestpeople interactions. Agriculture, Ecosystems and Environment 63:1-16.

Wiersum K.F. 1997b. From natural forest to tree crops, co-domestication of forest and tree species, an overview. Netherlands Journal of Agricultural Science 45:425-438.

Wong J.L.G., Thornber K. y Baker N. 2001. Evaluación de Recursos de Productos Forestales no Madereros. Experiencia y Principios Biométricos. Productos Forestales no Madereros 13. Fondo de las Naciones Unidas para la Alimentación y la Agricultura. Roma. 